\title{
Effects of zinc finger protein 403 on the proliferation, migration and invasion abilities of prostate cancer cells
}

\author{
XINTONG XU $^{1 *}$, ZHIHUI ZHU $^{1 *}$, YIPENG XU $^{2}$, SHASHA TIAN $^{1}$, YINGJUN JIANG ${ }^{2,3}$ and HUAJUN ZHAO ${ }^{1}$ \\ ${ }^{1}$ College of Pharmaceutical Sciences, Zhejiang Chinese Medical University, Hangzhou, Zhejiang 311402; \\ ${ }^{2}$ Institute of Cancer Research and Basic Medical Sciences of Chinese Academy of Sciences, Cancer Hospital of University of \\ Chinese Academy of Sciences, Zhejiang Cancer Hospital, Hangzhou, Zhejiang 310022; \\ ${ }^{3}$ The Second Clinical Medical College, Zhejiang Chinese Medical University, Hangzhou, Zhejiang 310053, P.R. China
}

Received January 22, 2020; Accepted August 19, 2020

DOI: $10.3892 / o r .2020 .7786$

\begin{abstract}
Zinc finger protein 403 (ZFP403), located on human chromosome $17 \mathrm{q} 12-21$, is closely associated with the development of cancer. However, to date, there are a limited number of studies on the biological functions of this gene, particularly in prostate cancer (PCa). The results of the present study demonstrated that compared with normal tissues, the expression of ZFP403 was markedly lower in PCa tissues, as shown by the evaluation of the Gene Expression Profiling Interactive Analysis 2 database. The decreased expression of ZFP403 in PCa clinical tissues and cell lines was confirmed by immunohistochemistry, reverse transcription-quantitative PCR and western blot analysis. Using short harpin (sh)RNA inhibition, stably-silenced ZFP403 cell lines were then constructed by lentiviral transfection (LV-PC3-shRNA-1 and 2; LV-DU145-shRNA-1 and 2). The results revealed that the knockdown of ZFP403 in PCa cells promoted cellular proliferation, colony formation, migration and invasiveness in vitro. Moreover, the levels of tumor growth- and motility-related proteins were significantly altered after ZFP403-knockdown. A xenograft tumor model using nude mice was established to elucidate the role of ZFP403 in tumorigenesis in vivo. Tumor growth was significantly increased in mice injected with ZFP403-knockdown cells compared with the control
\end{abstract}

Correspondence to: Dr Huajun Zhao, College of Pharmaceutical Sciences, Zhejiang Chinese Medical University, 260 Baichuan Street, Hangzhou, Zhejiang 311402, P.R. China

E-mail: zhj@zcmu.edu.cn

Dr Yipeng Xu, Institute of Cancer Research and Basic Medical Sciences of Chinese Academy of Sciences, Cancer Hospital of University of Chinese Academy of Sciences, Zhejiang Cancer Hospital, 38 Guangji Road, Hangzhou, Zhejiang 310022, P.R. China E-mail: xuyp1631@zjcc.org.cn

*Contributed equally

Key words: zinc finger protein 403, prostate cancer, growth, migration, invasion mice. Overall, the findings of the present study demonstrate that ZFP403 functions as a tumor suppressor gene in $\mathrm{PCa}$ by affecting the proliferation, migration and invasiveness of PCa cells, suggesting its potential use as a clinical diagnostic marker.

\section{Introduction}

Prostate cancer ( $\mathrm{PCa}$ ) is one of the most common malignant tumors of the male genitourinary system. According to pathological characteristics, the World Health Organization classifies PCa into five categories: i) adenocarcinoma (acinar adenocarcinoma); ii) ductal adenocarcinoma; iii) urothelial carcinoma; iv) squamous cell carcinoma; and v) and adenosquamous carcinoma, of which adenocarcinomas account for $>95 \%$ of cases. Current data indicate that PCa ranks second in incidence and fifth in mortality rate among male malignant tumors. Heterogeneity is one of the characteristics of $\mathrm{PCa}$, and it can be inert or highly invasive (1). In almost $90 \%$ of cases, $\mathrm{PCa}$ is confined to organs or locally advanced (2,3). However, $\mathrm{PCa}$ is aggressive and metastasizes through the blood and lymphatic systems, making it highly prone to recurrence in $10-15 \%$ of cases (4). Radiotherapy or radical prostatectomy are the preferred treatment methods for early-stage PCa, and are administered according to clinical stage and prostate-specific antigen levels (5). In highly metastatic PCa, antiandrogen therapy or androgen deprivation therapy (ADT) can reduce the levels of circulating testosterone by surgery or chemical castration $(3,6)$. However, these effects are short-lived, with the majority of patients becoming resistant to ADT after 18-36 months, and gradually developing castration-resistant PCa (CRPC) (2,7). Clinically, CRPC often exhibits a high degree of invasiveness and is associated with a poor response to treatment (8), and misdiagnosis leads to the unnecessary suffering of patients due to further treatments. Therefore, the identification of novel biomarkers that can be used to predict disease outcomes and promote the effective treatment of $\mathrm{PCa}$, is urgently required.

Zinc finger protein 403 (ZFP403), located in the 17q12-q21.1 region in humans, is highly conserved between Drosophila and humans. Mice and humans share $87 \%$ homology in their ZFP403 nucleotide sequences, and $96 \%$ homology in their 
amino acid sequences (9). In humans, ZFP403 has two known transcript types: full-length transcripts encoding a protein composed of 696 amino acids, known as gametogenetin binding protein 2 (GGNBP2), and short transcripts encoding a protein consisting of 288 amino acids, termed laryngeal cancer-related gene 1 (10-12).

ZFP403 is closely associated with the occurrence and development of several types of cancer (13). Studies have demonstrated that full-length ZFP403 may serve as a potential tumor suppressor, and that $Z F P 403$ is downregulated in various malignant tumors, such as breast (9), ovarian cancer (14) and glioma (15). Furthermore, the absence of ZFP403 can promote cellular proliferation (16) and tumorigenesis. However, the role of ZFP403 in PCa has not been fully investigated.

The aim of the present study was to determine the effect of ZFP403 on the carcinogenesis and progression of PCa, and to support the role of ZFP403 as a potential target for PCa by investigating its underlying mechanisms and effects on cell proliferation, migration and invasion therein.

\section{Materials and methods}

Differential expression analysis using the Gene Expression Profiling Interactive Analysis (GEPIA) database. Data from the GEPIA database (http://gepia.cancer-pku.cn/) were used to determine the association between ZFP403 and PCa. A gene symbol (ZFP403) was entered into the 'Enter gene name' field. The 'GoPIA!' button was clicked to generate the expression profile of the input gene across all tumor and normal tissues, in the form of dot plots or body maps. The 'Boxplot' tab in the 'Expression DIY' was clicked, and the 'PRAD' option was selected under 'Cancer name'; the results were then presented in box plots.

Tissue samples. PCa and adjacent tissues were collected from 19 male patients (aged 55 to 75 years old) who underwent treatment at the Department of Urology, Cancer Hospital of the University of Chinese Academy of Sciences (Hangzhou, China) between November 2013 and July 2015. The study was approved by the Hospital's Committee for the Protection of Human Subjects. All patients signed written informed consent forms in accordance with the Declaration of Helsinki.

Immunohistochemistry (IHC). IHC was used to determine the level of ZFP403 expression in clinical PCa tissues. The experiment was performed as previously described (14). The data were obtained by semi-quantitative analysis and finally presented as cut-off values. The cut-off values were determined by the multiplication of the staining intensity score and positive area score. Staining intensity scores were defined as follows: 0 , negative; 1 , weak; 2 , moderate; and 3 , strong. Positive area scores were defined as follows: $0,<25 \%$; $1,26-50 \% ; 2,51-75 \%$; and 3, $>75 \%$.

Cells and cell culture. The LNCaP, PC3, DU145, 22RV1 and RWPE-1 cell lines were purchased from the Chinese Academy of Sciences. Between December 5th and 12th, 2019, these cell lines were authenticated by the analysis of 21 autosomal short tandem repeat loci (Biowing Applied Biotechnology Co. Ltd.). RWPE-1 cells were cultured in K-SFM medium (Gibco;
Thermo Fisher Scientific, Inc.), and the other cell lines were maintained in DMEM (Gibco; Thermo Fisher Scientific, Inc.). DMEM contained 10\% fetal bovine serum (FBS; Gibco; Thermo Fisher Scientific, Inc.), and the cells were maintained at $37^{\circ} \mathrm{C}$ in a humidified atmosphere $\left(5 \% \mathrm{CO}_{2}\right)$.

Lentivirus infection and establishment of ZFP40-knockdown cells. Lentiviruses were purchased from Shanghai GeneChem. Short hairpin (sh)RNA sequences specifically targeting ZFP403 (shRNA1, 5'-GAGCAUACAAUAUCCUUAU-3'; shRNA2, 5'-GGGUAUUAGCAGAUUGGAA-3') were cloned into the hU6-MCS-CMV-Puromycin vector. An empty vector was used as the negative control. The cells were seeded $\left(1 \times 10^{5}\right)$ into a 24-well plate (Wuxi NEST Biotechnology Co., Ltd.) and cultured at $37^{\circ} \mathrm{C}$ overnight. Lentivirus $\left(1 \times 10^{8} \mathrm{TU} / \mathrm{ml}, 10 \mu \mathrm{l}\right)$ was mixed with Opti-MEM (500 $\mu \mathrm{l}$; Gibco; Thermo Fisher Scientific, Inc.) and polybrene (5 $\mu \mathrm{g} / \mathrm{ml}$; Shanghai GeneChem), and then used to infect the cells. The medium was replaced after $12 \mathrm{~h}$, and complete medium containing $1 \mu \mathrm{g} / \mathrm{ml}$ puromycin (Sigma-Aldrich; Merck KGaA) was used to establish cells in which ZFP403 had been stably silenced. After a week, the transfection efficiency was assessed by reverse transcription-quantitative PCR (RT-qPCR) and western blot.

Reverse transcription-quantitative (RT-q) PCR. The mRNA levels of ZFP403 in PCa cells were analyzed by RT-qPCR. According to the manufacturer's instructions, total RNA was extracted using TRIzol ${ }^{\circledR}$ (Invitrogen; Thermo Fisher Scientific, Inc.) and then reverse transcribed into cDNA using the PrimeScript ${ }^{\mathrm{TM}}$ RT reagent Kit (Takara Bio, Inc.). The concentration of nucleic acid was quantified using a cell Imaging Multi-mode Reader (BioTek Instruments, Inc.). Subsequently, qPCR was performed with SYBR-Green (Bio-Rad Laboratories, Inc.) using the CFX96 Real-Time system (Bio-Rad Laboratories, Inc.). The thermocycling parameters were as follows: $95^{\circ} \mathrm{C}$ for $2 \mathrm{~min} ; 40$ cycles at $95^{\circ} \mathrm{C}$ for $20 \mathrm{sec}, 58^{\circ} \mathrm{C}$ for $20 \mathrm{sec}$, and $72^{\circ} \mathrm{C}$ for $15 \mathrm{sec}$; from $65^{\circ} \mathrm{C}$ to $95^{\circ} \mathrm{C}$, to rise $0.5^{\circ} \mathrm{C}$ every $5 \mathrm{sec}$. The relative expression level of ZFP403 was quantified using the $2^{-\Delta \Delta C q}$ method (17). The specific primer sequences were as previously described: ZFP403 forward, 5'-ACAGGGTATTAGCAGATTGGAAC-3' and reverse, 5'-TCATTGGTAACAATTACTTCTACAC-3'; and GAPDH forward, 5'-AGAAGGCTGGGGCTCATTTG-3' and reverse, 5'-AGGGGCCATCCACAGTCTTC-3' (14).

Western blotanalysis. Total protein was extracted fromPCacells using RIPA lysis buffer (Beyotime Institute of Biotechnology) and the concentration of protein was determined using a BCA Protein Assay kit (Beyotime Institute of Biotechnology). The samples ( $50 \mu \mathrm{g}$ per lane) were separated by 8 or $10 \%$ SDS-PAGE and then transferred onto PVDF membranes (EMD Millipore). After blocking with TBST containing 5\% skim milk at room temperature for $1 \mathrm{~h}$, the membranes were incubated overnight at $4{ }^{\circ} \mathrm{C}$ with the following primary antibodies as appropriate: GGNBP2 (1:250; cat. no. ab203104, Sigma-Aldrich; Merck KGaA); cdc2 (cat. no. 9116), cyclin B1 (cat. no. 12231), $\beta$-catenin (cat. no. 9562), MMP-2 (cat. no. 4022) and $\beta$-actin (cat. no. 4970) (all 1:1,000; all from Cell Signaling Technology, Inc.); cdc25kC (1:500; cat. no. sc-13138; Santa Cruz Biotechnology, Inc.); E-cadherin (cat. no. 1702-1) and 
vimentin (cat. no. 2862-1) (1:1,000; both from Epitomics, Inc.); and heparanase (1;500; cat. no. ab42817; Abcam). Goat anti-rabbit IgG $(\mathrm{H}+\mathrm{L})$-HRP and goat anti-mouse IgG $(\mathrm{H}+\mathrm{L})$-HRP (1:3,000; Bio-Rad Laboratories, Inc.) were used as secondary antibodies and incubated with the membrane at room temperature for $2 \mathrm{~h}$. Protein bands were visualized using Clarity $^{\mathrm{TM}}$ Western ECL Substrate (Bio-Rad Laboratories, Inc.) (18).

Colony formation assay. In vitro colony formation ability and tumorigenicity were investigated by plate and soft-agar colony formation assays. For plate colony formation assays, 500 cells per well were seeded into 24 -well plates and cultured at $37^{\circ} \mathrm{C}$. After 14 days, the cells were fixed with $4 \%$ paraformaldehyde for $15 \mathrm{~min}$, and then stained with freshly prepared crystal violet for $20 \mathrm{~min}$ (both at room temperature). For the soft-agar colony formation assay, cells $\left(1 \times 10^{3}\right.$ per well) were plated in $0.35 \%$ low melting point agarose above a solidified $0.7 \%$-agarose layer in a 24 -well plate. The cells were cultured at $37^{\circ} \mathrm{C}$ and the medium was changed twice a week. After 28 days, $1 \mathrm{mg} / \mathrm{ml}$ iodonitrotetrazolium chloride (Sigma-Aldrich; Merck KGaA) was added to each well and the plate was incubated for a further $24 \mathrm{~h}$. The colonies were counted under a Leica DM500 microscope (magnification, x40; Leica Microsystems, Inc.) (14).

Cell cycle analysis. The cells were stained using the PI/RNase staining kit (BD Pharmingen; BD Biosciences), and the distribution of the cell cycle was analyzed by flow cytometry using the Guava easyCyte 6HT-2L (Merck KGaA) (19). The results were analyzed using ModFit LT $^{\mathrm{TM}}$ software (Windows version 4.0; Verity Software House).

Migration and invasion assays. The migratory and invasive abilities of PCa cells were investigated using a Transwell chamber (Corning, Inc.). For invasion assays, Matrigel (BD Biosciences) was thawed at $4^{\circ} \mathrm{C}$ overnight and diluted in serum-free medium. Then, $50 \mu \mathrm{l}$ diluted Matrigel was added to the upper chamber and incubated at $37^{\circ} \mathrm{C}$ for $20 \mathrm{~min}$. Cells $\left(1 \times 10^{5}\right)$ were seeded into the upper chamber with serum-free medium containing $0.5 \%$ BSA, while the lower chamber was filled with $600 \mu \mathrm{l}$ medium containing $10 \% \mathrm{FBS}$. After incubation for $24 \mathrm{~h}$ at $37^{\circ} \mathrm{C}$, the cells were fixed with $4 \%$ polyoxymethylene for $15 \mathrm{~min}$ at room temperature, and then stained using the Hematoxylin and Eosin Staining Kit (Beyotime Institute of Biotechnology). The cells that had not passed through the polycarbonate membrane were removed with a cotton swab, and the invasive cells were visualized using a Leica DM500 microscope and counted in five randomly selected fields (magnification, x100). For migration assays, all conditions were consistent, except for the use of Matrigel (20).

Xenograft tumor study. Male BALB/c nude mice $(\mathrm{n}=12)$ were obtained from the Shanghai Laboratory Animal Center (CAS), and were used at 5 weeks of age. The mice were housed and maintained in specific pathogen-free conditions under a $12 \mathrm{~h}$ light-dark cycle at $25^{\circ} \mathrm{C}$, with free access to food and water. The tumor xenograft experiments were approved and conducted according to the guidelines provided by the Experimental Animal Center of Zhejiang Chinese Medical University (no. SYXK-2018-0012). PC3 cells with or without ZFP403-knockdown were harvested, resuspended in serum-free DMEM medium $\left(2 \times 10^{6}\right)$ and subcutaneously injected into the dorsa of the mice (21). Animal health and behavior were monitored daily. When the tumor burden had reached $\sim 50 \mathrm{~mm}^{3}$, the sizes were measured every 3 days and the tumor volume was calculated using the following formula: $\mathrm{V}=1 / 2$ (length $\mathrm{x}$ width ${ }^{2}$ ). After 40 days, the mice were sacrificed by cervical dislocation. Death was verified by the absence of a heartbeat and the onset of rigor mortis.

Statistical analysis. The data are presented as the mean \pm SD. Comparison between multiple groups was performed using one-way ANOVA followed by Tukey's post hoc test, while a paired Student's t-test was used for comparisons between 2 groups. All data analyses were performed using GraphPad Prism version 5 (GraphPad Software, Inc.), and $\mathrm{P}<0.05$ was considered to indicate a statistically significant difference.

\section{Results}

ZFP403 is downregulated in human PCa. Data from the GEPIA2) database were used to determine the association between ZFP403 and PCa. Differential gene expression analysis revealed that $Z F P 403$ expression was lower in $\mathrm{PCa}$ tissues than in normal tissues (Fig. 1A). To further examine the expression level of ZFP403 in PCa, 19 groups of PCa and corresponding adjacent tissues were analyzed using IHC. The results revealed that the expression of ZFP403 in PCa tissues was significantly lower than that in adjacent normal tissues $(\mathrm{P}<0.01$; Fig. 1B and C). RT-qPCR and western blot analysis were then used to detect the ZFP403 level in normal prostatic epithelial cells (RWPE-1) and PCa cell lines (LNCaP, PC3, DU145 and 22RV1). As shown in Fig. 1D and E, the expression levels of ZFP403 were lower in cancer cells than in RWPE-1 cells $(\mathrm{P}<0.01)$. These data indicate that ZFP403 may function as a tumor suppressor in $\mathrm{PCa}$.

ZFP403-knockdown promotes the proliferation of $\mathrm{PCa}$ cells. To evaluate the role of ZFP403 in the proliferation PCa cells, human PCa cell lines (PC3 and DU145), which are insensitive to androgens, were selected for transfection with ZFP403-shRNA lentivirus. The results revealed that the expression of $Z F P 403$ was markedly decreased in the ZFP403-knockdown cells compared with that in the negative control (NC) cells, though to greater degree in cells transfected with ZFP403-shRNA-1 (Fig. 2A and B). Plate colony formation assays were performed to determine the effects of ZFP403 on colony formation. The results demonstrated that knocking down ZFP403 significantly increased PC3 and DU145 cell colony numbers (Fig. 2C). Furthermore, in the soft-agar colony formation assay, ZFP403-knockdown resulted in a marked increase in the number of colonies (Fig. 2D), indicating that ZFP403 inhibits tumorigenicity in vitro.

In order to further examine the effect of ZFP403 on the proliferation of $\mathrm{PCa}$ cells, the cell cycle distribution was detected by flow cytometry. The results revealed that the number of cells transitioning from the $\mathrm{S}$ phase to the $\mathrm{G}_{2} / \mathrm{M}$ phase was significantly increased in PCa cells in which 
A

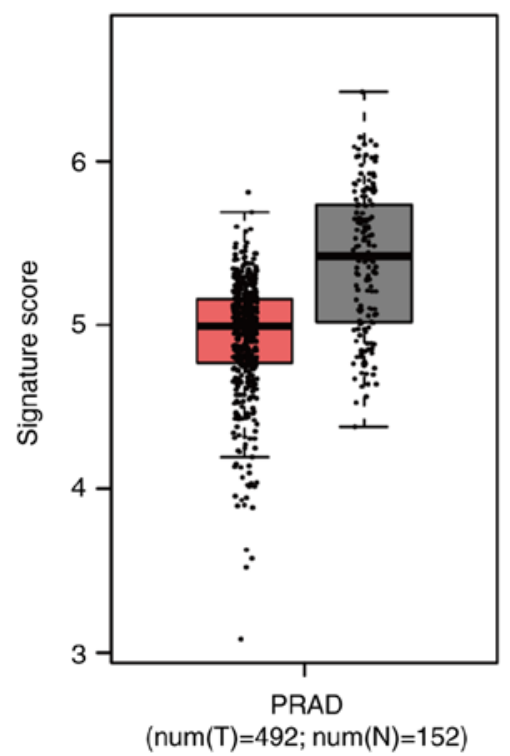

C

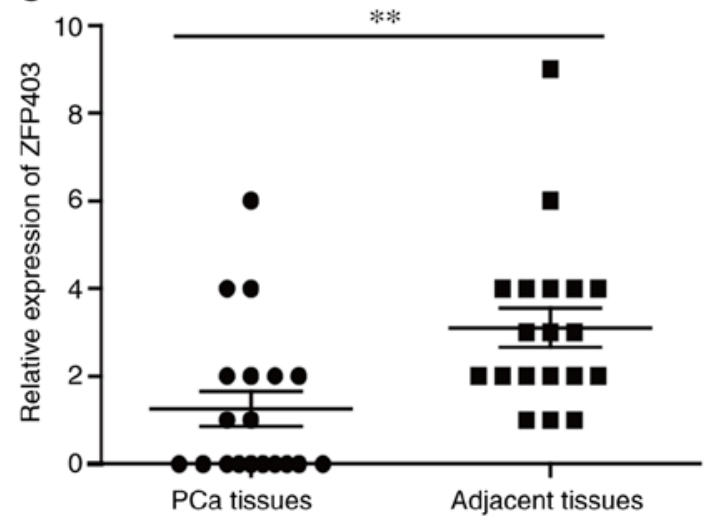

B

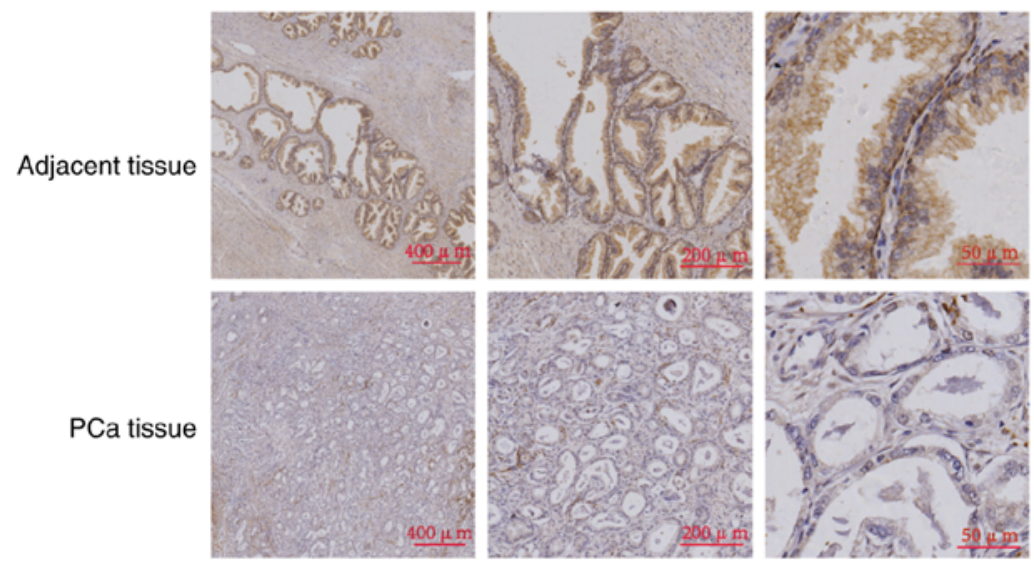

D

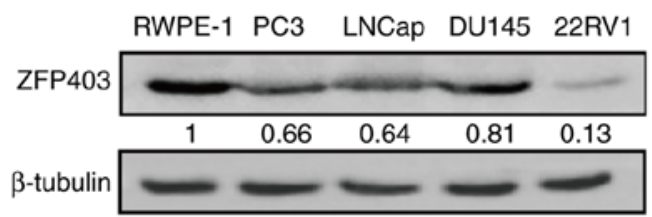

E

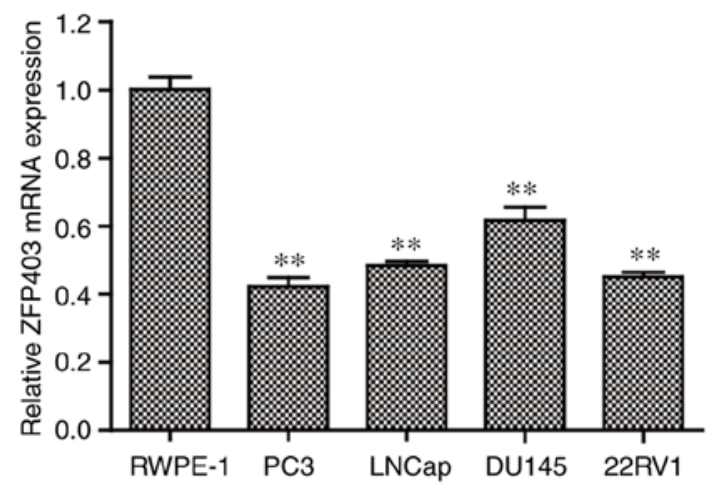

Figure 1. Expression of ZFP403 in human PCa tissues and cell lines. (A) Box plots of signature score analysis using the Gene Expression Profiling Interactive Analysis 2 database, calculated as the mean value of $\log 2(T P M+1)$. Tumor samples are indicated by the red box, while the gray box represents normal tissues. (B) Expression levels of ZFP403 protein in PCa tissues and paired adjacent normal tissues, detected by IHC. (C) Expression of ZFP403 quantified according to the cut-off value (a cut-off value $\geq 3$ indicates positive expression) for the IHC results. Relative expression levels of $Z F P 403$ (D) protein and (E) mRNA were determined by reverse transcription-quantitative PCR and western blot analyses. ${ }^{* *} \mathrm{P}<0.01$. ZFP403, Zinc finger protein 403 ; PCa, prostate cancer; IHC, immunohistochemistry; PRAD, prostate adenocarcinoma; T, tumor; N, normal.

ZFP403 was knocked down (Fig. 3A and B). On this basis, western blot analysis was performed to detect the expression of $\mathrm{G}_{2} / \mathrm{M}$ phase-related proteins. The results demonstrated that knocking down ZFP403 upregulated the expression of cyclin B1, cdc2 and cdc25C (Fig. 3C). These data indicate that silencing ZFP403 retains the viability and promotes the proliferation of PCa cells.

ZFP403-knockdown promotes the migration and invasion abilities of $P C$ a cells. To determine the potential role of ZFP4O3 in the metastasis of PCa, the effects of ZPF403 on migration and invasion were evaluated using Transwell chamber migration and invasion assays. The results revealed that the cell migratory and invasive abilities were significantly promoted following ZFP403-knockdown (Fig. 4A and B).

Furtherexperiments demonstrated thatZFP403-knockdown in PC3 and DU145 cells resulted in downregulation of the epithelial cell marker E-cadherin, and the upregulation of the interstitial cell marker vimentin. In addition, $\beta$-catenin, heparanase and matrix metalloproteinase 2 (MMP2) were also upregulated, as shown in Fig. 4C. Taken together, these results suggest that silencing ZFP403 promotes PCa metastasis by enhancing cell migration and invasiveness.

Antitumor effect of ZFP403 on tumor xenografts in vivo. In order to examine the effect of ZFP403 on tumor development and progression in vivo, a tumor xenograft model of PCa cells was established by subcutaneous inoculation of $\mathrm{BALB} / \mathrm{c}$ nude mice. Body weight and tumor volume were measured every 3 days for 40 days. The growth rate of subcutaneous tumors in the ZFP403-knockdown group was significantly higher than that in the NC group. However, no significant difference in body weight was observed between the groups (Fig. 5A-D). In addition, compared with the 
A

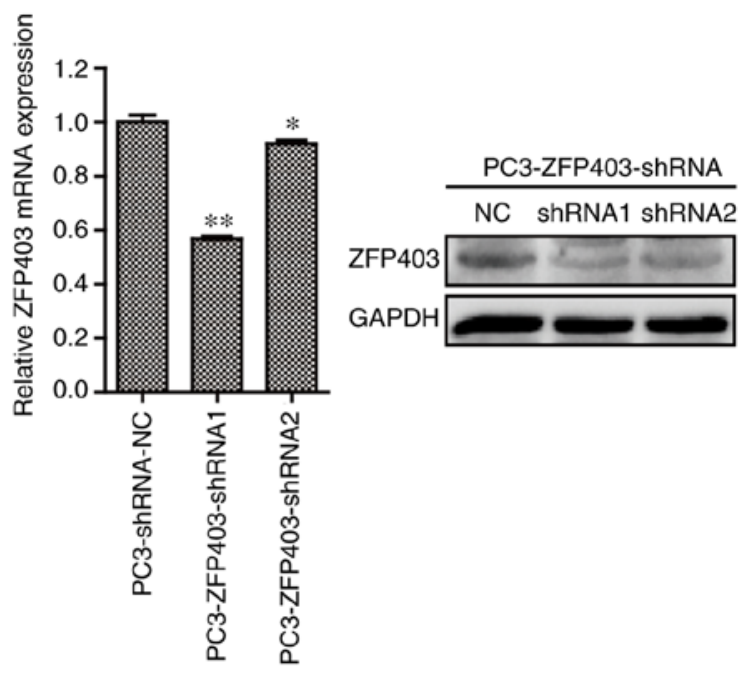

B

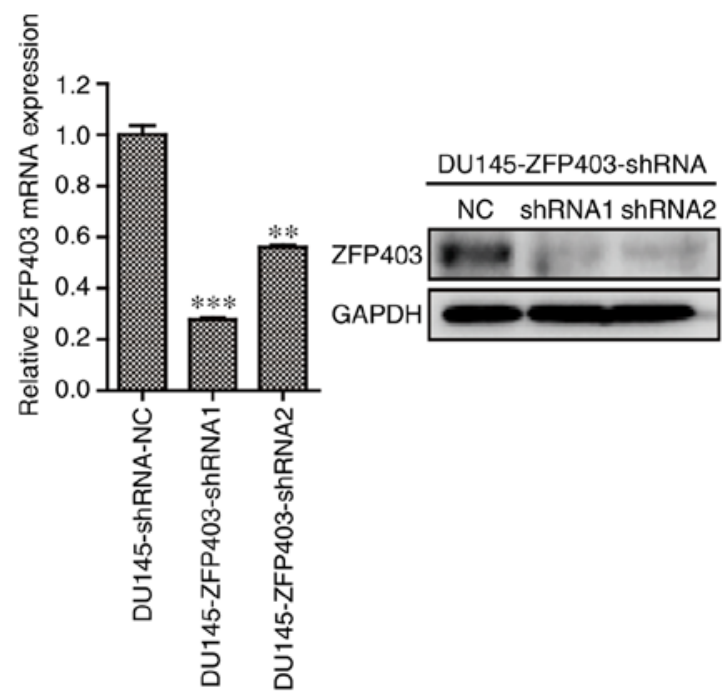

C

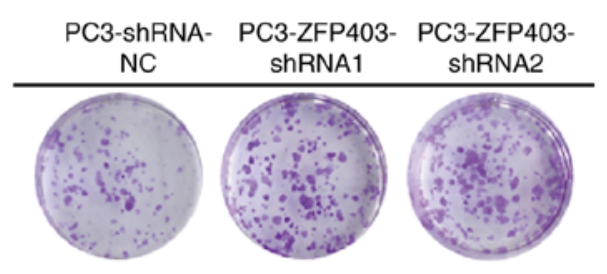

DU145-shRNA- DU145-ZFP403- DU145-ZFP403NC ShRNA1 ShRNA2

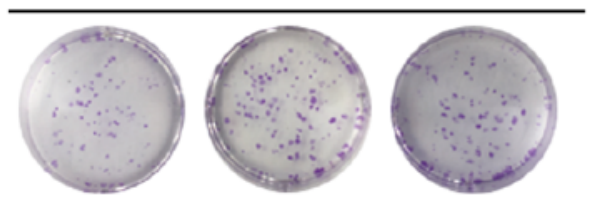

D

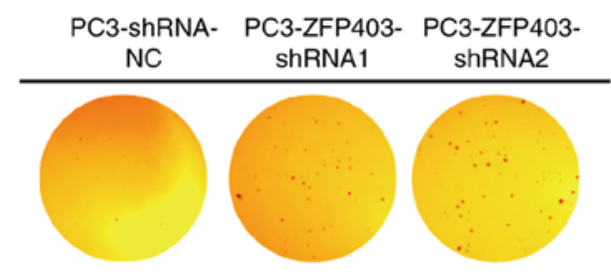

DU145-shRNA- DU145-ZFP403- DU145-ZFP403NC ShRNA1
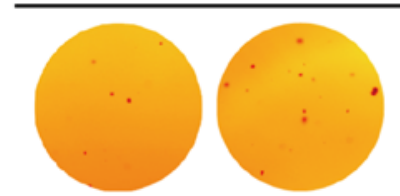
ShRNA2

Figure 2. Effects of ZFP403 on the proliferation of PC3 and DU145 cells. Reverse transcription-quantitative PCR and western blot analyses were performed to assess the efficiency of ZFP403-knockdown in (A) PC3 and (B) DU145 cells. (C) Plate colony formation and (D) soft-agar colony formation assays were performed to determine the effects of ZFP403-knockdown on PC3 and DU145 cells. Colony numbers were counted and images were captured. Results represent the mean $\pm \mathrm{SD}$ from three independent experiments. ${ }^{*} \mathrm{P}<0.05,{ }^{* *} \mathrm{P}<0.01$ and ${ }^{* * * *} \mathrm{P}<0.001$ vs. the NC control. ZFP403, Zinc finger protein 403 ; PCa, prostate cancer; sh(RNA), short hairpin; NC, negative control.

NC group, the mRNA and protein levels of ZFP403 were decreased in the ZFP403-knockdown group, as shown by
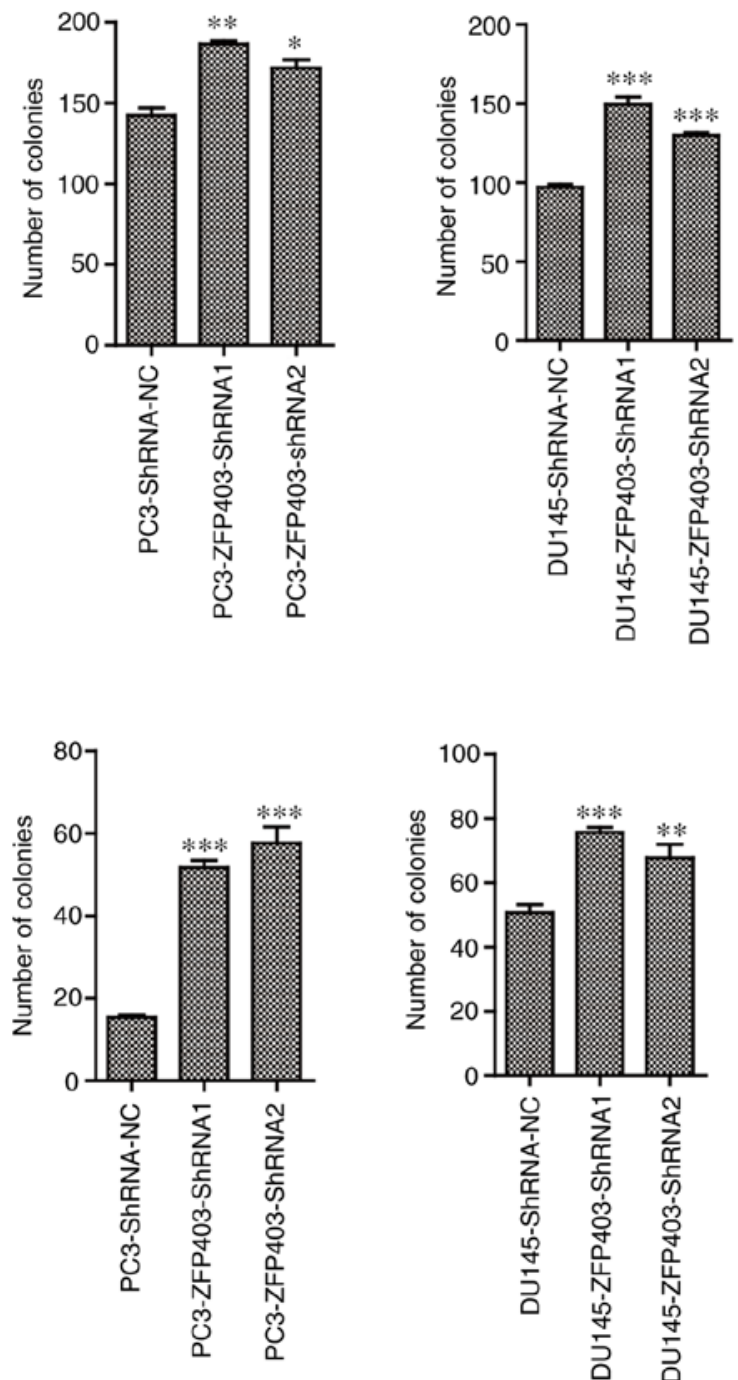

RT-qPCR and western blot analysis, respectively (Fig. 5E-F). These data suggest that decreased ZFP403 expression is 


\section{A PC3-shRNA-NC PC3-ZFP403-shRNA1 PC3-ZFP403-shRNA2}
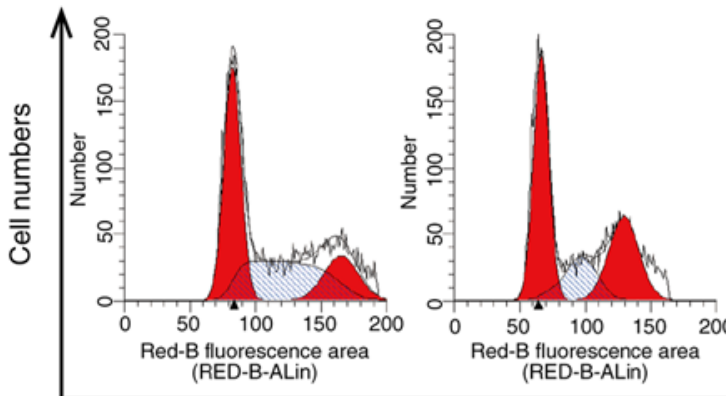
(RED-B-ALin)

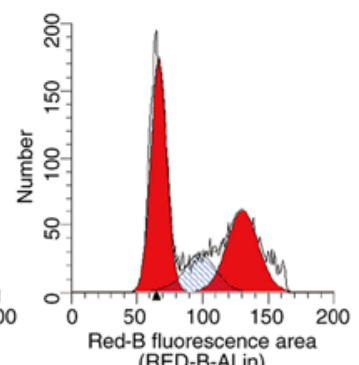
(RED-B-ALin)

B DU145-shRNA-NC DU145-ZFP403-shRNA1 DU145-ZFP403-shRNA2
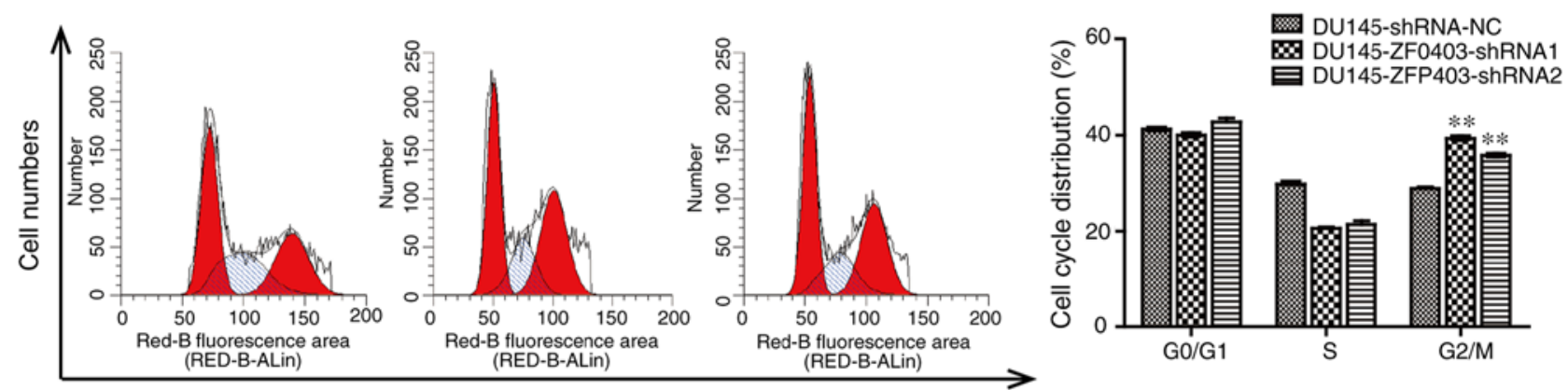
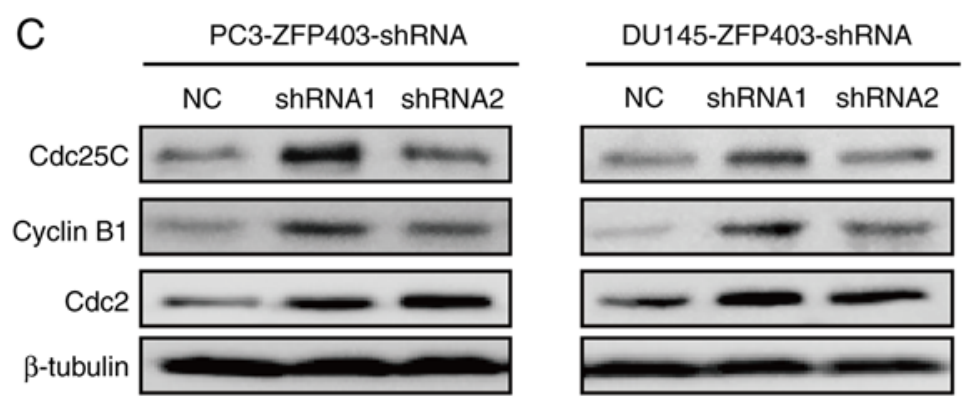

Figure 3. ZFP403-knockdown induces cell cycle arrest at the $\mathrm{G}_{2} / \mathrm{M}$ phase. PI staining was used to detect the effects of ZFP403 on the cell cycle, using flow cytometric analysis in (A) PC3 and (B) DU145 cells. (C) Protein levels of critical $\mathrm{G}_{2} / \mathrm{M}$-phase regulators were detected by western blot analysis. The data are presented as the mean \pm SD from three independent experiments. ${ }^{* *} \mathrm{P}<0.01$ and ${ }^{* * * *} \mathrm{P}<0.001$ vs. the NC control. ZFP403, Zinc finger protein 403; sh(RNA), short hairpin; NC, negative control.

closely associated with the tumorigenicity and development of PCa in vivo.

\section{Discussion}

Due to the aging of the population and changes in lifestyle, PCa has gradually become one of the most common cancer types among men worldwide $(22,23)$. Currently, radical prostatectomy, radiotherapy and hormone therapy are effective options for the treatment of early-stage $\mathrm{PCa}$; however, there are still challenges for the clinical treatment of advanced $\mathrm{PCa}(24)$. Anti-androgen therapy is usually effective in $80-90 \%$ of patients with advanced or aggressive $\mathrm{PCa}$, and has become the standard treatment of choice (25). However, ADT is only effective against androgen-dependent $\mathrm{PCa}$, and does not eliminate androgen-sensitive or androgen-independent cancer. Therefore, the majority of patients enter a hormone-insensitive state after a few years of hormone therapy, and eventually fail to respond to hormone therapy $(26,27)$. In addition, radiotherapy and chemotherapy are largely ineffective in patients with advanced PCa, and may even result in severe toxicity and side-effects $(28,29)$.

With the development of molecular biological technologies, and further understanding of the mechanisms of tumorigenesis at the cellular and molecular levels, the targeted molecular therapy of tumors has entered a new era. Worldwide, $>60 \%$ of ongoing clinical trials of molecular targeted therapy are for cancer, including brain, lung, breast, pancreatic, liver, colorectal, bladder, head and neck, skin, ovarian and kidney cancer, as well as PCa (30). Targeted anticancer drugs commonly used in clinical practice include Herceptin, Glivec, Iressa and Tagrisso (31). However, few of these drugs are applicable for the treatment of $\mathrm{PCa}$, thus the identification of novel potential targets for $\mathrm{PCa}$ is critical.

In the present study, in order to further determine the progression of patients with advanced and castration-resistant PCa, androgen-independent PCa cells (PC3 and DU145) (32) were used to investigate the functions of ZFP403. The expression of ZFP403 in PCa tissues was significantly lower than that in adjacent tissues. Furthermore, the expression of ZFP403 at 
A

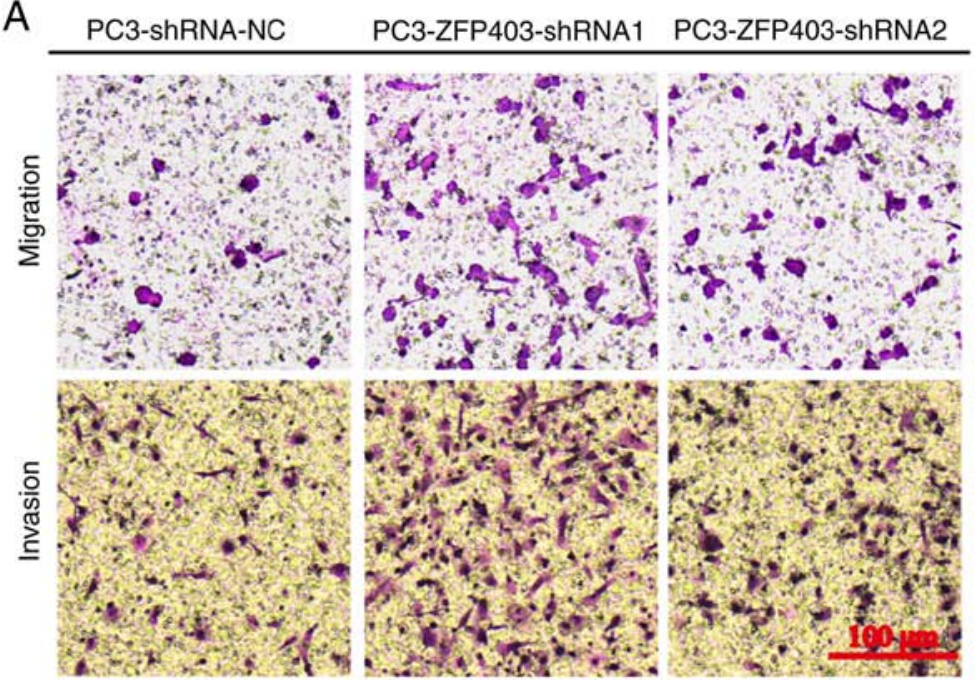

B

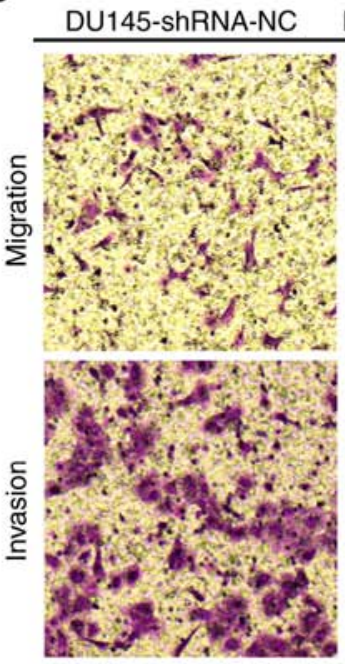

DU145-ZFP403-shRNA1 DU145-ZFP403-shRNA1
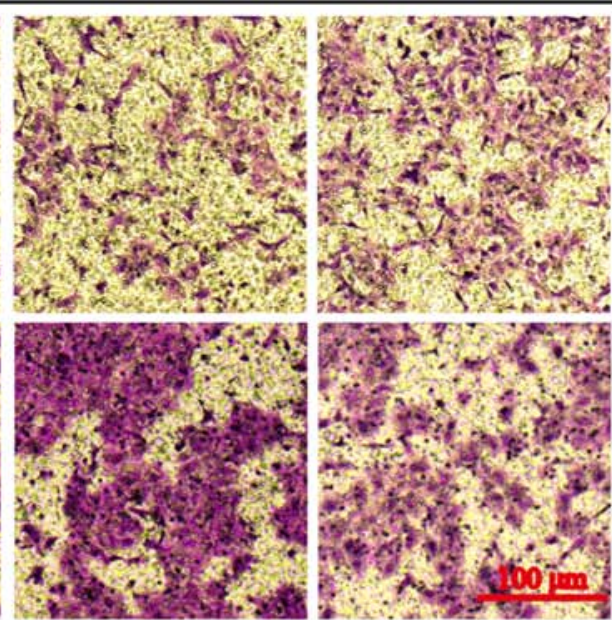

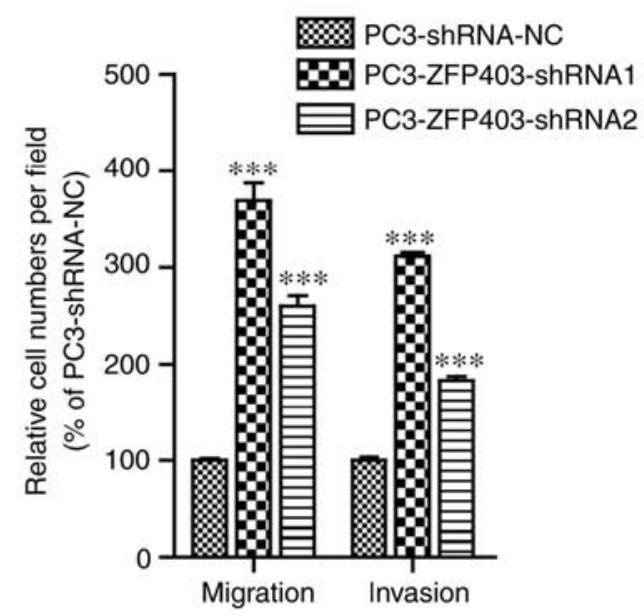

C
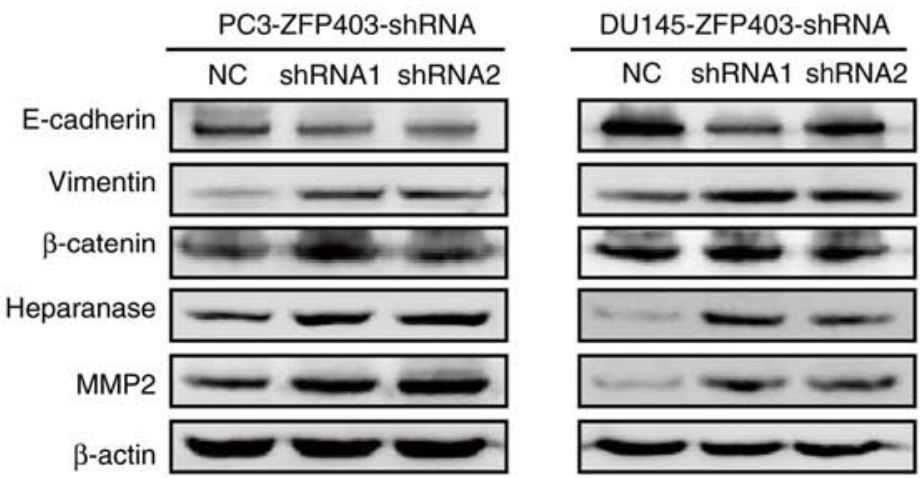

Figure 4. ZFP403-knockdown promotes the migration and invasion abilities of PC3 and DU145 cells. Transwell migration and Matrigel invasion assays were performed to confirm that ZFP403-shRNA inhibits the migratory and invasive abilities of (A) PC3 and (B) DU145 cells. (C) Western blot analysis was used to detect relative protein levels in ZFP403-knockdown cells compared with NC cells. All data are presented as the mean \pm SD from three independent experiments. ${ }^{* * *} \mathrm{P}<0.001$ vs. the NC control. ZFP403, Zinc finger protein 403; sh(RNA), short hairpin; NC, negative control; MMP2, matrix metalloproteinase 2.

both the protein and mRNA level in different PCa cells was lower than that in normal epithelial prostate cells, which was consistent with the aforementioned IHC results. The decreased expression of ZFP403 maintained the growth of $\mathrm{PCa}$, indicating that ZFP403 may be involved in the progression of $\mathrm{PCa}$ as a tumor suppressor gene. shRNA interference and lentivirus packaging technology were then used to silence ZFP403 for further analysis.
To further investigate the biological functions of $Z F P 403$, the effects of ZFP403-knockdown on the proliferation and metastasis of PCa cells were evaluated. The results revealed that in vitro cell colony formation ability and tumorigenicity were enhanced following the knockdown of ZFP403. The cell cycle is one of the key modes of regulating cell growth, thus the effect of ZFP403 on cell cycle distribution was examined by flow cytometry. The results demonstrated that 
A

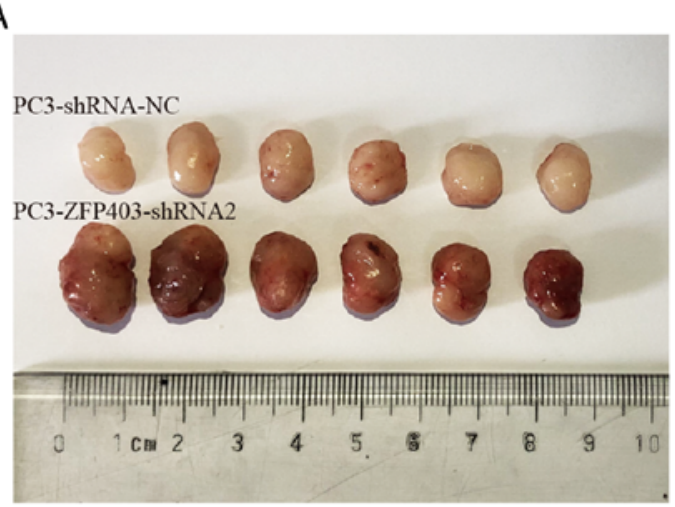

C

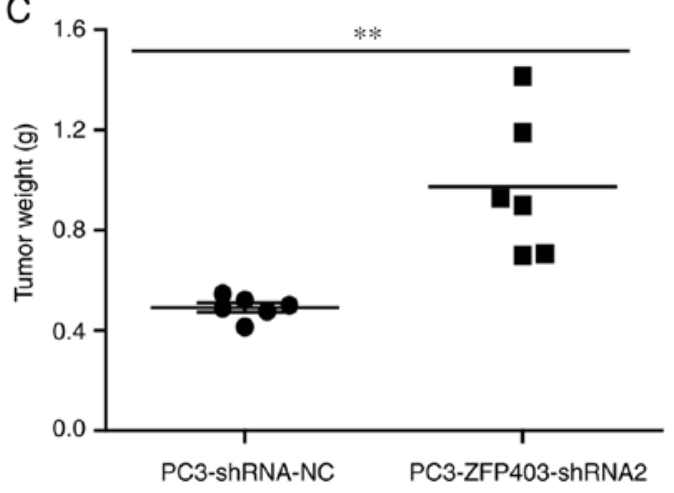

E

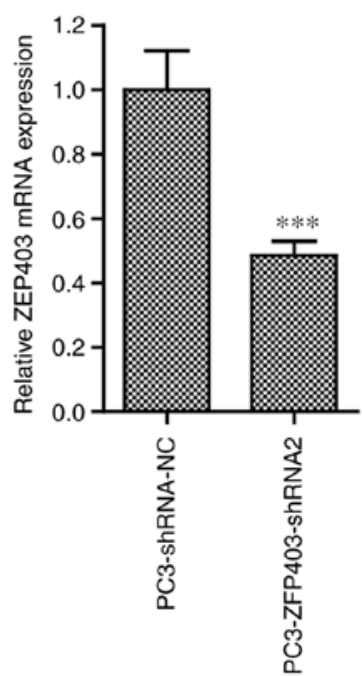

B

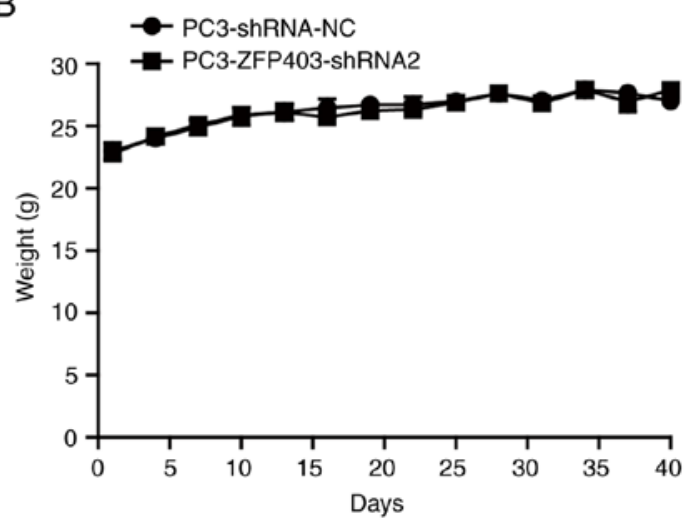

D

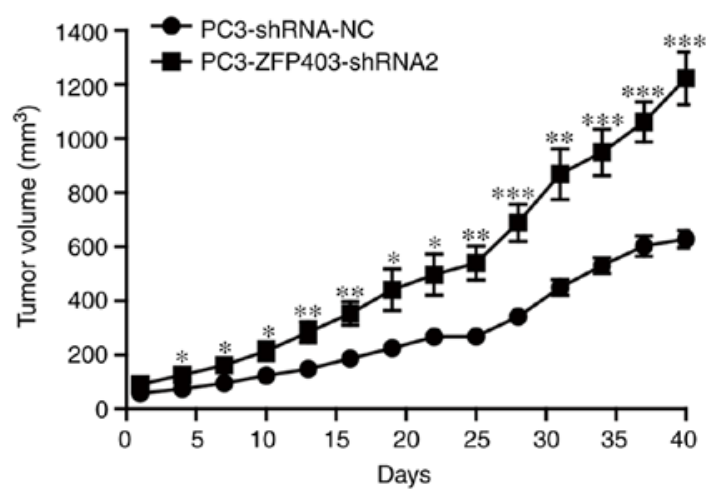

$\mathrm{F}$

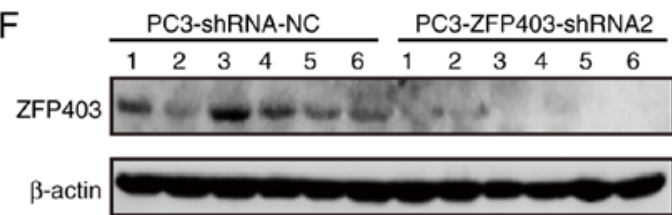

Figure 5. ZFP403-knockdown promotes the tumorigenesis of PC3 cells in vivo. (A) A xenograft model of PCa was established via the subcutaneous injection of PC3-ZFP403-shRNA and PC3-NC cells into the dorsa of nude mice (n=6/group). After 40 days of measurement, the mice were sacrificed and the tumors were dissected and photographed. (B) Body weight and (D) tumor growth volumes were measured every 3 days. (C) Final tumor weights were measured. (E) Reverse transcription-quantitative PCR and (F) western blot analyses were performed to detect the expression levels of ZFP403 in different tumor tissues. Results are presented as the mean $\pm \mathrm{SD} .{ }^{*} \mathrm{P}<0.05,{ }^{* *} \mathrm{P}<0.01$ and ${ }^{* * *} \mathrm{P}<0.001$ vs. the NC control. ZFP403, Zinc finger protein 403 ; PCa, prostate cancer; sh(RNA), short hairpin; NC, negative control.

the number of cells transitioning from the $\mathrm{S}$ to the $\mathrm{G}_{2} / \mathrm{M}$ phase was increased in ZFP403-knockdown cells compared with NC cells.

Cell cycle progression is regulated by cyclin-dependent kinase, whose activity is strictly regulated by cyclin. The ectopic expression of cyclin is associated with the progression of a number of malignant tumors (33). Studies have indicated that the expression of cyclin B1 plays a key role in the $\mathrm{G}_{2} / \mathrm{M}$-phase transition of human PCa cells $(34,35)$. Studies using the transgenic PCa mouse model have also demonstrated that the expression of cyclin B1 is increased in poorly-differentiated androgen-independent $\mathrm{PCa}$ (36). Thus, in the present study, cyclin-related proteins were assessed in the $\mathrm{G}_{2} / \mathrm{M}$ phase before and after ZFP403-knockdown. The results revealed that the expression levels of cdc2 and cyclin B1 were upregulated, while the expression of cdc25C was downregulated, suggesting that ZFP403-knockdown accelerates the transition to the $\mathrm{G}_{2} / \mathrm{M}$ phase and promotes cancer cell proliferation. However, the presence of phosphorylated cdc25C could not be detected (data not shown). This result is consistent with the 
upregulation of cdc25C expression in $\mathrm{PCa}$ tissues, primarily in the form of dephosphorylation (33).

ZFP403 is a classical Cys2His2 (C2H2)-type zinc finger protein, which is encoded by $2 \%$ of human genes (37), constituting the largest sequence-specific DNA binding protein family (38). Numerous studies have demonstrated that $\mathrm{C} 2 \mathrm{H} 2$-type zinc finger proteins can regulate the transcription of downstream genes involved in cellular proliferation and metastasis. At the same time, $\mathrm{C} 2 \mathrm{H} 2$-type zinc finger proteins act as recruiters of chromatin modifiers or structural proteins, regulating the migration and invasion abilities of cancer cells $(39,40)$. Therefore, it was hypothesized that ZFP403 may be involved in the migration and invasiveness of PCa. Indeed, the results of Transwell migration and invasion assays confirmed this hypothesis, where ZFP403-knockdown significantly enhanced the migration and invasion capacities of PCa cells. Further experiments also demonstrated that $\mathrm{ZFP} 403$-knockdown promoted metastasis by regulating the expression of epithelial markers, mesenchymal markers, MMP2, heparanase and $\beta$-catenin. $\beta$-catenin and E-cadherin are usually present as an E-cadherin $/ \beta$-catenin complex located in cell-cell adherent junctions in the cell membrane. However, the loss of E-cadherin leads to epithelial-mesenchymal transition (EMT), accompanied by the deregulation of the Wnt signaling pathway. In addition, as a key component of the Wnt pathway, $\beta$-catenin plays an important role in the negative regulation of E-cadherin and EMT $(41,42)$. Research has indicated that disassociation of the E-cadherin/ $\beta$-catenin complex leads to the suppression of E-cadherin and the nuclear translocation of $\beta$-catenin, which enhances the invasive and migratory potential of tumors (42).

In order to improve our understanding of the function of ZFP403 in tumor development and progression in vivo, a xenograft model was used in the present study. ZFP403-knockdown induced the formation and development of transplanted tumors in nude mice, suggesting that ZFP403 may be a potential tumor suppressor in $\mathrm{PCa}$.

In conclusion, the effect of ZFP403 in PCa was preliminarily examined in the present study. The results demonstrate that ZFP403-knockdown promotes the progression of $\mathrm{PCa}$ by enhancing proliferation, migration and invasiveness. Furthermore, ZFP403 was confirmed to function as a tumor suppressor in $\mathrm{PCa}$, and this finding is consistent with the results of the overexpression of ZFP403 in PC3 (43). Future studies will aim to further investigate the functions of $Z F P 403$ in $\mathrm{PCa}$, in order to establish a novel therapeutic target for patients with advanced metastatic and hormone-independent PCa.

\section{Acknowledgements}

Not applicable.

\section{Funding}

The present study was supported by the Natural Science Foundation of China (grant no. 81774003), the Zhejiang Provincial Natural Science Foundation of China (grant no. LY16H160034) and the Zhejiang Basic Public Welfare Research Projects (grant no. LQ19H160006).

\section{Availability of data and materials}

The datasets used and/or analyzed during the current study are available from the corresponding author on reasonable request.

\section{Authors' contributions}

$\mathrm{XX}$ and $\mathrm{ZZ}$ performed the experiments and contributed to the study design, as well as the acquisition, analysis and interpretation of the data. YX and YJ collected and analyzed the clinical samples. ST contributed to the acquisition, analysis and interpretation of the data. HZ contributed to study conception and revised the manuscript critically. All authors have read and approved the final manuscript.

\section{Ethics approval and consent to participate}

The present study was approved by the Ethics Committee of Zhejiang Cancer Hospital (Hangzhou, China), and all enrolled patients provided written informed consent. The animal experiments were conducted with the approval of the Experimental Animal Ethical Committee of Zhejiang Chinese Medical University (SYXK20180012).

\section{Patient consent for publication}

No applicable.

\section{Competing interests}

The authors declare that they have no competing interests.

\section{References}

1. Vlaeminck-Guillem V, Gillet G and Rimokh R: SRC: Marker or actor in prostate cancer aggressiveness. Front Oncol 4: 222, 2014.

2. Yap TA, Smith AD, Ferraldeschi R, Al-Lazikani B, Workman P and de Bono JS: Drug discovery in advanced prostate cancer: Translating biology into therapy. Nat Rev Drug Discov 15: 699-718, 2016.

3. Nevedomskaya E, Baumgart SJ and Haendler B: Recent advances in prostate cancer treatment and drug discovery. Int J Mol Sci 19: 1359,2018

4. Haymart MR, Miller DC and Hawley ST: Active surveillance for low-risk cancers-a viable solution to overtreatment? N Engl J Med 377: 203-206, 2017.

5. Crawford ED, Heidenreich A, Lawrentschuk N, Tombal B, Pompeo ACL, Mendoza-Valdes A, Miller K, Debruyne FMJ and Klotz L: Androgen-targeted therapy in men with prostate cancer: Evolving practice and future considerations. Prostate Cancer Prostatic Dis 22: 24-38, 2019.

6. Pan TF, Liang CZ, Chen XG and Fan S: Mammalian target of rapamycin regulates androgen receptor and Akt phosphorylation in prostate cancer 22RV1 cells. Zhonghua Nan Ke Xue 19: 1068-1071, 2013 (In Chinese).

7. Sumanasuriya $S$ and De Bono J: Treatment of advanced prostate cancer-a review of current therapies and future promise. Cold Spring Harb Perspect Med 8: a030635, 2018.

8. Wu CT, Chen WC and Chen MF: The response of prostate cancer to androgen deprivation and irradiation due to immune modulation. Cancers (Basel) 11: 20, 2018.

9. Lan ZJ, Hu YH, Zhang S, Li X, Zhou H, Ding J, Klinge CM, Radde BN, Cooney AJ, Zhang J and Lei Z: GGNBP2 acts as a tumor suppressor by inhibiting estrogen receptor $\alpha$ activity in breast cancer cells. Breast Cancer Res Treat 158: 263-276, 2016. 
10. Guan R, Wen XY, Wu J, Duan R, Cao H, Lam S, Hou D, Wang Y, $\mathrm{Hu} \mathrm{J}$ and Chen Z: Knockdown of ZNF403 inhibits cell proliferation and induces $\mathrm{G} 2 / \mathrm{M}$ arrest by modulating cell-cycle mediators. Mol Cell Biochem 365: 211-222, 2012.

11. Zhang J, Wang Y, Zhou Y, Cao Z, Huang P and Lu B: Yeast two-hybrid screens imply that GGNBP1, GGNBP2 and OAZ3 are potential interaction partners of testicular germ cell-specific protein GGN1. FEBS Lett 579: 559-566, 2005.

12. Li Y and Chen $\mathrm{Z}$ : Molecular cloning and characterization of LCRG1 a novel gene localized to the tumor suppressor locus D17S800-D17S930. Cancer Lett 209: 75-85, 2004.

13. Plummer SJ, Paris MJ, Myles J, Tubbs R, Crowe J and Casey G: Four regions of allelic imbalance on 17q12-qter associated with high-grade breast tumors. Genes Chromosomes Cancer 20: 354-362, 1997.

14. Zhu Z, Lou C, Zheng Z, Zhu R, Tian S, Xie C and Zhao H: ZFP403, a novel tumor suppressor, inhibits the proliferation and metastasis in ovarian cancer. Gynecol Oncol 147: 418-425, 2017.

15. Zhan A, Lei B, Wu H, Wen YT, Zheng L, Wang S, Wan X and Wei Z: GGNBP2 suppresses the proliferation, invasion, and migration of human glioma cells. Oncol Res 25: 831-842, 2017.

16. Guo K, He Y, Liu L, Liang Z, Li X, Cai L, Lan ZJ, Zhou J, Wang $\mathrm{H}$ and Lei Z: Ablation of Ggnbp2 impairs meiotic DNA double-strand break repair during spermatogenesis in mice. J Cell Mol Med 22: 4863-4874, 2018.

17. Livak KJ and Schmittgen TD: Analysis of relative gene expression data using real-time quantitative PCR and the 2(-Delta Delta C(T)) method. Methods 25: 402-408, 2001.

18. Yang B, Zhao Y, Lou C and Zhao H: Eupalinolide O, a novel sesquiterpene lactone from eupatorium lindleyanum DC. induces cell cycle arrest and apoptosis in human MDA-MB-468 breast cancer cells. Oncol Rep 36: 2807-2813, 2016.

19. Tian SS, Chen Y, Yang B, Lou C, Zhu R, Zhao Y and Zhao H: F1012-2 inhibits the growth of triple negative breast cancer through induction of cell cycle arrest, apoptosis, and autophagy. Phytother Res 32: 908-922, 2018.

20. Li LZ, Zhang CZ, Liu LL, Yi C, Lu SX, Zhou X, Zhang ZJ, Peng YH, Yang YZ and Yun JP: miR-720 inhibits tumor invasion and migration in breast cancer by targeting TWIST1. Carcinogenesis 35: 469-478, 2014.

21. Yang B,Zhu R, Tian S, Wang Y,Lou S and ZhaoH: Jatamanvaltrate $\mathrm{P}$ induces cell cycle arrest, apoptosis and autophagy in human breast cancer cells in vitro and in vivo. Biomed Pharmacother 89: 1027-1036, 2017.

22. Baade PD, Youlden DR and Krnjacki LJ: International epidemiology of prostate cancer: Geographical distribution and secular trends. Mol Nutr Food Res 53: 171-184, 2009.

23. Torre LA, Bray F, Siegel RL, Ferlay J, Lortet-Tieulent J and Jemal A: Global cancer statistics, 2012. CA Cancer J Clin 65 $87-108,2015$

24. Chang AJ, Autio KA, Roach M III and Scher HI: High-risk prostate cancer-classification and therapy. Nat Rev Clin Oncol 11: 308-323, 2014.

25. Miyamoto H, Messing EM and Chang C: Androgen deprivation therapy for prostate cancer: Current status and future prospects. Prostate 61: 332-353, 2004.

26. Uemura $H$, Ishiguro $H$, Nakaigawa $N$, Nagashima $Y$, Miyoshi $Y$, Fujinami K, Sakaguchi A and Kubota Y: Angiotensin II receptor blocker shows antiproliferative activity in prostate cancer cells: A possibility of tyrosine kinase inhibitor of growth factor. Mol Cancer Ther 2: 1139-1147, 2003.
27. Li J, Luo J, Gu D, Jie F, Pei N, Li A, Chen X, Zhang Y, Du H, Chen B, et al: Adenovirus-mediated angiotensin II type 2 receptor overexpression inhibits tumor growth of prostate cancer in vivo. J Cancer 7: 184-191, 2016.

28. Walsh PC, DeWeese TL and Eisenberger MA: Clinical practice. Localized prostate cancer. N Engl J Med 357: 2696-2705, 2007.

29. Bommareddy A, Rule B, VanWert AL, Santha S and Dwivedi C: $\alpha$-Santalol, a derivative of sandalwood oil, induces apoptosis in human prostate cancer cells by causing caspase- 3 activation. Phytomedicine 19: 804-811, 2012.

30. Wirth T and Ylä-Herttuala S: Gene therapy used in cancer treatment. Biomedicines 2: 149-162, 2014.

31. Workman P: New drug targets for genomic cancer therapy: Successes, limitations, opportunities and future challenges. Curr Cancer Drug Targets 1: 33-47, 2001.

32. Saraon P, Musrap N, Cretu D, Karagiannis GS, Batruch I, Smith C, Drabovich AP, Trudel D, van der Kwast T, Morrissey C, et al: Proteomic profiling of androgen-independent prostate cancer cell lines reveals a role for protein $\mathrm{S}$ during the development of high grade and castration-resistant prostate cancer. J Biol Chem 287: 34019-34031, 2012.

33. Ozen $M$ and Ittmann $M$ : Increased expression and activity of CDC25C phosphatase and an alternatively spliced variant in prostate cancer. Clin Cancer Res 11: 4701-4706, 2005.

34. Mashal RD, Lester S, Corless C, Richie JP, Chandra R, Propert KJ and Dutta A: Expression of cell cycle-regulated proteins in prostate cancer. Cancer Res 56: 4159-4163, 1996.

35. Kallakury BV, Sheehan CE, Ambros RA, Fisher HA, Kaufman RP Jr, Muraca PJ and Ross JS: Correlation of p34cdc2 cyclin-dependent kinase overexpression, CD44s downregulation, and HER-2/neu oncogene amplification with recurrence in prostatic adenocarcinomas. J Clin Oncol 16: 1302-1309, 1998

36. Maddison LA, Huss WJ, Barrios RM and Greenberg NM: Differential expression of cell cycle regulatory molecules and evidence for a 'cyclin switch' during progression of prostate cancer. Prostate 58: 335-344, 2004.

37. Lander ES, Linton LM, Birren B, Nusbaum C, Zody MC, Baldwin J, Devon K, Dewar K, Doyle M, FitzHugh W, et al: Initial sequencing and analysis of the human genome. Nature 409: 860-921, 2001.

38. Tupler R, Perini G and Green MR: Expressing the human genome. Nature 409: 832-833, 2001.

39. Jen JY and Wang YC: Zinc finger proteins in cancer progression. J Biomed Sci 23: 53, 2016.

40. Cassandri M, Smirnov A, Novelli F, Pitolli C, Agostini M, Malewicz M, Melino G and Raschellà G: Zinc-finger proteins in health and disease. Cell Death Discov 3: 17017, 2017.

41. Clarke NW, Hart CA and Brown MD: Molecular mechanisms of metastasis in prostate cancer. Asian J Androl 11: 57-67, 2009.

42. Chen HN, Yuan K, Xie N, Wang K, Huang Z, Chen Y, Dou Q, Wu M, Nice EC, Zhou ZG and Huang C: PDLIM1 stabilizes the E-Cadherin/ $\beta$-catenin complex to prevent epithelial-mesenchymal transition and metastatic potential of colorectal cancer cells. Cancer Res 76: 1122-1134, 2016.

43. Yang Z, Wang Y and Ma L: Effects of gametogenetin-binding protein 2 on proliferation, invasion and migration of prostate cancer PC-3 cells. Andrologia 52: e13488, 2020.

This work is licensed under a Creative Commons Attribution-NonCommercial-NoDerivatives 4.0 International (CC BY-NC-ND 4.0) License. 